\title{
Targeted drug delivery to the virus-infected airway; complications and remedies.
}

\author{
Daniel T. W. Clarke and Nigel A. J. McMillan* \\ Griffith Health Institute and School of Medical Science, Griffith University, Gold \\ Coast, Australia.
}

${ }^{*}$ Corresponding author

email: n.mcmillan@griffith.edu.au

Phone +61755 527135

Fax +61755528908

Running Title: Drug delivery to the airway.

Keywords: Airway, drug delivery, infection, inhalation, therapeutics, virus. 


\begin{abstract}
Drug delivery to the airway and lower respiratory tract by aerosol inhalation has become a successful, non-invasive method of preventing and treating local disease of the lung. Consequently, it has been a promising route for clinical trials using highly specific and novel therapies to overcome viral pulmonary infection such as RNA interference, neutralising monoclonal antibodies and microparticle treatments. Yet despite this great potential, this form of delivery has proven somewhat ineffective due to airway remodeling, inflammation and mucus hypersecretion that results from viral symptoms in the respiratory tract. Here we review the research into the delivery technologies available as well as the types of therapeutics used for respiratory virus disease and examine how virus infection-induced airway inflammation modulates its success. We discuss the future of aerosol administration and present potential alternative methods for efficient drug delivery so as to improve post-infection virus control therapies.
\end{abstract}

\title{
Introduction
}

Growing attention has been given to the potential of the pulmonary route as a non-invasive means of delivery of therapeutic agents ever since the invention of liquid nebulisers around the turn of the 19th century. From the notions of what once were ancient remedies to treat disease, such as inhaling burning leaves or the vapours of aromatic plants, came the first legitimate pharmaceutical therapies. In 1925, inhaled insulin began to be administered in experimental studies of diabetes [1], and in the 1940s, an inhaled formulation of epinephrine was marketed with hand-bulb nebulisers for the treatment of asthma [2]. This was further refined in 1955 when the president of Riker Labs developed a pressurised metered dose inhaler (MDI) at the suggestion of his asthmatic daughter who noticed the mechanism behind perfume spray devices [3]. More recently, advances in molecule design, drug discovery and delivery technologies have facilitated the expansion of various systems, formulations and devices to 
administer drugs both locally for the treatment of diseases of the respiratory tract as well as systemically via the lung.

The respiratory system is susceptible to a range of diverse diseases such as asthma, cystic fibrosis, emphysema, pulmonary arterial hypertension and chronic obstructive pulmonary disease. Additionally, due to its location and physiological function, the pulmonary system is the primary site of entry and establishment of many airborne pathogens, particularly viruses. Six viruses are the main causative agents of localised respiratory illness: rhinovirus, respiratory syncytial virus (RSV), influenza virus, parainfluenza virus (PIV), corona virus, and adenovirus. Infections with these respiratory viruses not only cause symptomatic disease, but can also exacerbate pre-existing conditions such as bronchial asthma $[4,5]$. The human papillomavirus has also been shown to infect the mucosal surfaces of the upper respiratory tract causing respiratory papillomatosis [6], which in rare cases can lead to airway obstruction [7] or progress to head and neck carcinomas [8]. Furthermore, viruses such as enterovirus, measles and hanta virus are known to cause disseminated disease following entry via the lung. Consequently, these are all prime targets for treatment with pulmonary delivery systems. Despite a number of recent advances showing great promise to treat these viral infections, the pulmonary delivery of peptides and proteins can be impeded by the complexity of the anatomic structure of the human respiratory system. This is further complicated in the diseased state as delivery sites can be obscured by excess mucus secretion in the upper respiratory tract and tissue remodeling from an anti-viral inflammatory response. In this review we will outline the current technologies and molecules used to overcome viral disease, examine the delivery routes and barriers encountered with these therapies during viral infection and offer potential alternatives for efficient drug delivery.

\section{Delivery technologies}

The selection of delivery device plays an important role in the formulation 
design and overall success of the system. As the required particle/droplet size depends on the specific target location within the respiratory tract, the selected device must be capable of generating and delivering stable compounds of the desired aerosolised particle diameter. The devices most commonly used for respiratory delivery are shown in figure 1 and include nebulisers, aerosolisers, metered dose inhalers (MDIs) and dry powder inhalers (DPIs).

Nebuliser (air jet)

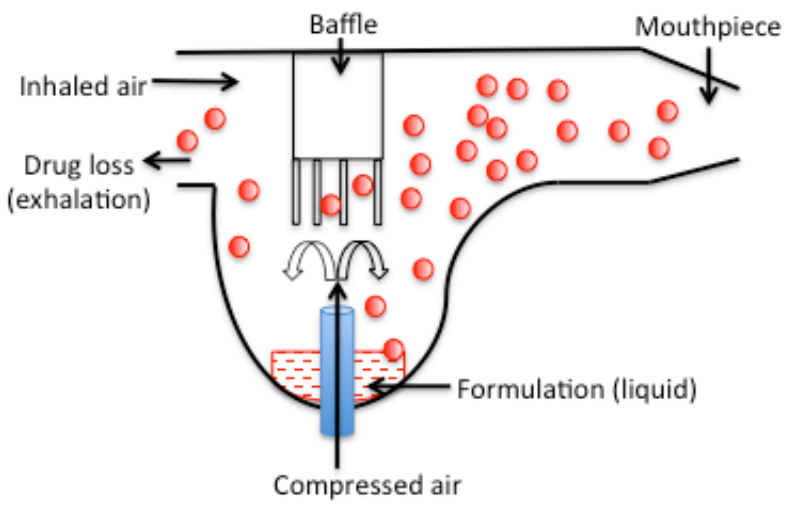

Metered dose inhaler

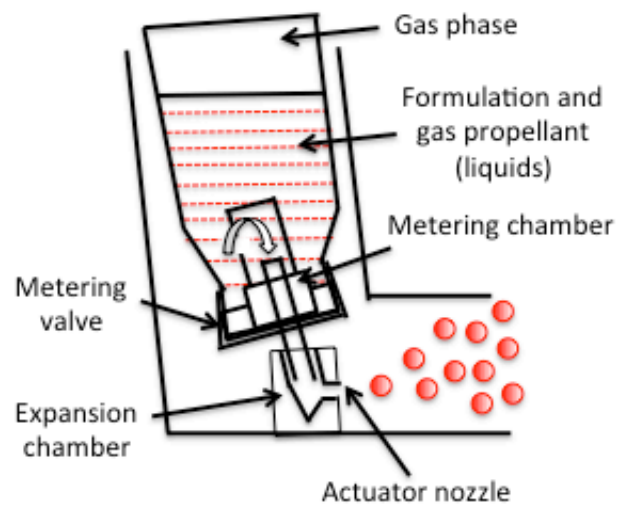

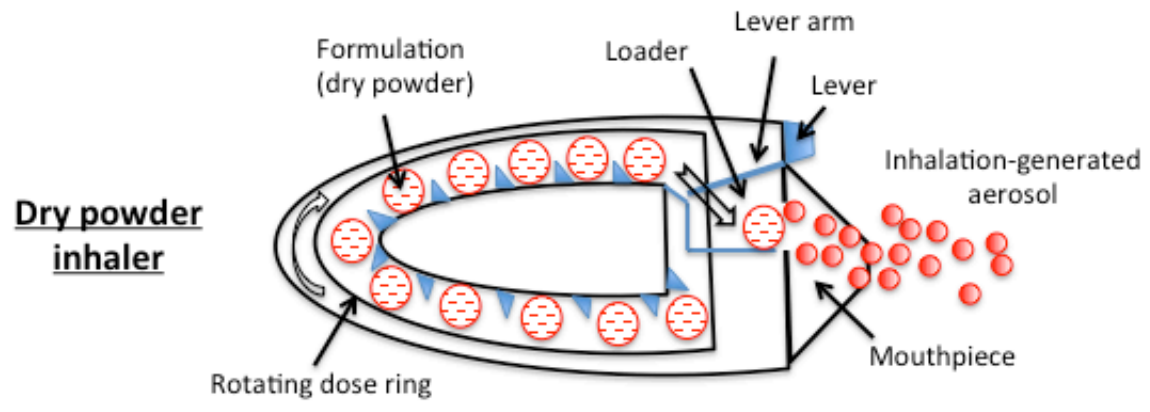

Figure 1. Mechanism of action of various drug delivery technologies used to deliver compounds to the respiratory tract. Pulmonary drugs can be administered using devices that rely on compressed air (nebuliser), propellant (MDIs), or inhalation (DPIs) for delivery.

The majority of anti-viral therapy studies to date have often used conventional jet nebulisers to generate small particle aerosols in the hope of delivering drug compounds directly to the site of viral replication and limit systemic exposure. 
One of the first ever anti-viral drugs discovered, ribavirin (or Virazole), was approved in 1986 for the treatment of severe RSV infections by nebuliser delivery [9]. These devices administer atomised, aqueous drug solution by air jet or ultrasonic mechanisms at a continuous rate over multiple inhalations and are often used for critical or ambulatory care. There are several types of nebulisers available such as jet nebulisers that are driven by compressed air, ultrasonic nebulisers that use a transducer to create droplets from a liquid reservoir, or vibrating mesh nebulisers that vibrate to generate fine particles. Similarly, alternative aerosolisers may use propellant to quickly and efficiently administer drug product concentrate directly via spray, often in a manner that has reduced irritation when compared to mechanical application. They have proven successful in delivering a number of therapeutic compounds to the respiratory tract with good efficacy and appear to be well tolerated. Compliance to this form of treatment is usually easily achieved and the direct delivery is especially important to reduce viral load in immunocompromised patients or those with viruses resistant to other forms of therapy such as oral oseltamivir in the case of influenza [10]. Continual development is underway on alternative aerosol delivery devices and this could lead to better distribution, mucous penetration and overall uptake efficiency in pulmonary epithelial cells.

Another device that produces an aerosol from liquid drug formulations is the MDI. These are comparable to nebulisers, but instead expel exact measured amounts of preformed medication mixed with propellant upon activation. This allows for the delivery of accurate doses of the compound, with minimal wastage, from a convenient and portable tool. Due to the ease of selfadministration, adherence to treatment regimes can be less than ideal, however monitoring accessories are continually being developed to improve outcomes, such as the SmartTrack device [11]. In the past, MDIs have had more of a role in the treatment of asthma or chronic obstructive pulmonary disease as anti-viral treatments have favoured the use of nebulisers. Consequently, use of these devices tends to have been restricted to testing post-virus treatments, such as administering corticosteroids following RSV infection to improve recurrent wheeze [12]. Recently, MDIs have been instrumental in the development of 
combination therapies where identical deposition patterns of separate compounds can be achieved from the one dose [13]. Therefore they may prove useful in delivering anti-viral compounds and steroidal drugs concurrently to ameliorate disease outcomes in the future.

Lastly, the DPI devices have been the most popular instrument used to deliver drugs, particularly proteins to the lungs. These devices use micronised medication blended with carrier (but no propellant) and requires the patient to inhale the powder for efficient delivery. The way some blends behave and the interaction of powders with the device have been unclear in the past and computational fluid dynamics have helped to improve design and development of DPI devices. One such study demonstrated how device retention and overall inhaler performance could be improved by altering grid structure and mouthpiece length [14]. These types of improvements have led to a number of successful handheld tools, such as the Diskhaler, which has been widely used to deliver the influenza drug zanamivir (or Relenza). Using this device has demonstrated significant reductions in the median time to alleviate major influenza symptoms by up to 2.5 days compared with placebo in phase III trials [15] and are likely to be used for future anti-viral therapies.

\section{Therapeutic molecules}

While a great deal of recent research has focused on the development of novel delivery devices, growing efforts are being dedicated to the various formulations of macromolecular drugs. As the pulmonary membrane is naturally permeable to small molecules, peptides and proteins, many of the first studies attempted direct delivery of naked oligonucleotides or proteins into the respiratory tract. These compounds are thought to be absorbed rapidly into the body as drug efflux transporters and metabolising enzymes are present at low levels, especially when compared to other sites such as the gastrointestinal tract. However, in the case of naked oligonucleotides such as short-interfering RNA (siRNA), the bio-distribution throughout the lung and the uptake mechanism 
remains unclear. The negative charge and size of these molecules would suggest that they are not able to bind to the cell surface or cross the cell membrane via passive mechanisms and may explain in part why naked siRNA has not been particularly effective in early clinical trials for RSV [16]. Consequently, effective delivery has been investigated using carrier molecules or vectors to transport drug cargo to the cell (figure 2).

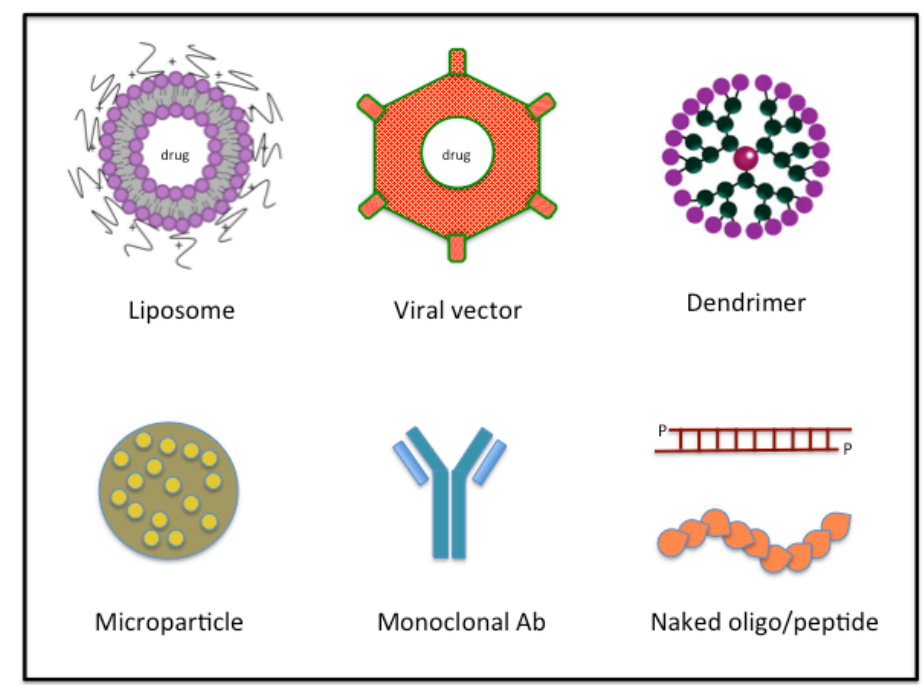

Figure 2. Different therapeutic compounds used for delivery to the respiratory tract. Pulmonary drugs can range from naked nucleic acids and peptides to conjugated carrier particles or viral vectors.

Vaccination therapies have been important in viral prophylaxis and a number of research groups have delivered live attenuated virus to generate viral-specific immunity [17-20]. This idea has been adapted to use viral vectors such as adenoviruses and lentiviruses to deliver therapeutic small RNAs into the respiratory system to treat inflammatory disease [21]. Despite some success due to efficient delivery via viral vectors to target cells in the lung, their immunogenicity limits their broad or repeated use. Innate immune responses to viruses not only impede gene delivery but can also cause severe complications for the patient. In light of these concerns, non-viral delivery has emerged as a promising alternative for the delivery of therapeutic effectors.

The success of drug delivery can be reduced by enzymes in the lung that diminish overall bioavailability, and by the barrier between cells and alveolar air. 
Therefore absorption enhancers and carrier vectors have been examined to increase the pulmonary absorption of macromolecular or poorly absorbable drugs [22]. By exploiting their inherent properties, such as hydrophilicity, lipophilicity or net negative charge, these compounds can be complexed to positively charged lipids, fusion proteins or polymers to enhance interactions with the cell surface. Examples of these carrier systems include liposomes, microparticles and dendrimers.

Liposomes are small vesicles consisting of an aqueous core surrounded by one or more natural phospholipids that form a closed bilayer. They can act as carriers for both lipophilic and hydrophilic drugs and have a high degree of biocompatibility and diversity. These structures became a popular tool following the observation that complexing compounds with cationic lipid could improve delivery to cells. Tompkins and colleagues demonstrated protection from lethal infection with influenza virus in mice by transporting unmodified siRNA specific to influenza nucleoprotein and polymerase genes using the cationic lipid Oligofectamine [23]. A similar outcome was achieved against RSV by using siRNAs targeting the phosphoprotein gene that were linked to a commercial transfection lipid [24]. The study also showed an improved outcome over using naked siRNA alone, with pulmonary viral titres being reduced only by $70 \%$ of the complexed siRNA treatment in mice.

These results have led to a number of further studies including liposomal delivery of peptides, oligonucleotides, chemicals and anti-viral drugs. Vaccine approaches have been developed using liposomes to deliver short, highly conserved influenza-derived peptides to the respiratory tract to elicit potent innate and selective adaptive immune responses [25]. Oligonucleotides such as viral-specific siRNAs [26] and double-stranded RNA immune modulators [27] have been packaged into liposome carriers to be efficiently delivered to cells in order to protect against lethal challenge with influenza virus either by RNA interference or stimulation of innate immunity. The influenza drug oseltamivir (or TamiFlu), which requires large oral doses and is associated with anti-viral resistance, has been investigated for the development of dry powder liposome 
formulations for intranasal administration, offering the opportunity to reduce dosing frequency [28]. Liposomal-encapsulated chemicals have also been used as a tool to examine the role of immune cells in viral respiratory disease. As it became apparent that alveolar macrophages played a pivotal role in inflammation and immunostimulation, the intranasal inoculation of clodronate complexed within liposomes has been used to deplete these cells and examine the effects. Mice treated this way before immunisation against RSV showed markedly reduced interstitial inflammation, but also developed lower levels of serum RSV-neutralising antibody and impaired protection against RSV challenge [29]. Alternatively, this treatment prior to infection with the severe acute respiratory syndrome coronavirus (SARS-CoV) showed that severe disease could be overcome as the alveolar macrophages had an inhibitory effect, preventing the development of immune responses [30]. Liposomal delivery this way has allowed localised examination without affecting the level of circulating macrophages and could help to tease out essential mechanisms involved in disease.

Another carrier particle similar to liposomes is micro- or nano-particles. These are hollow, spherical carriers that range from 0.1 to 500 microns in size and instead of lipids, usually consist of a polysaccharide outer layer such as chitosan, which is mucoadhesive, biodegradable, biocompatible and proven safe for human use [31]. Zhang and colleagues demonstrated its application by using the nanochitosan polymer NG042 to deliver a siRNA plasmid targeting the NS1 gene of RSV into the lungs and inhibit infection [32]. It also proved effective in reducing virus titres in the lung and prevented inflammation associated with infection in rats [33].

Another potential carrier used to transport therapeutic compounds is dendrimers. These are hyperbranched structures used to encapsulate peptides or saccharides and tend to be 10 to 100 nanometres in diameter. They have been shown to be efficient as gene delivery vectors [34], with polyamidoamine dendrimer-complexed plasmid DNA producing significant levels of gene expression in the murine lung [35]. Previous work has shown that intranasal 
administration of dendrimers carrying sialic acid was able to prevent adhesion of various influenza subtypes in a murine influenza pneumonitis model and inhibit infection [36]. More recently, there have been few examples of this technology used in the treatment of viruses. However, higher generation dendrimers have been shown to be highly biocompatible in vivo and more efficient at transfecting cultured cells [37] and so this could lead to effective delivery in the future.

Other than viral vectors and non-viral carrier systems, humanised antibodies have become prevalent as an anti-viral therapy, either alone or in combination with other compounds. However, so far they have only been administered by intramuscular or intravenous injection and have not been adequately tested via inhalation. Prevention of RSV infection was first observed from a prophylactic polyclonal immune globulin and this allowed for the development of palivizumab, a neutralising monoclonal antibody directed against the fusion protein of RSV [38]. It was first seen to produce a 99\% reduction of lung RSV titres and has been used clinically to treat serious RSV-related respiratory disease, especially in high-risk infants. After extensive use, it was discovered that a small number of patients were not adequately protected and this has given rise to motavizumab, an affinity-optimized version of palivizumab with improved protection against RSV in vivo $[39,40]$. The enhanced ability of motavizumab to prevent RSV infection is being evaluated in multiple human clinical trials, but to date, a single intravenous dose of the antibody does not appear to decrease viral load or severity of illness following RSV infection [41].

Neutralising antibodies have also been investigated for the treatment of influenza but due to the hypervariability in the amino-acid sequence of its viral surface antigens, efficacy can be quite low, especially among different groups and subtypes. An antibody isolated many years ago from a mouse immunised with the H2N2 virus was found to cross-neutralise a number of $\mathrm{H}$ subtypes [42] yet little progress has been made until recently. In 2011, the passive transfer of a monoclonal antibody selected from plasma cells was shown to recognise all $\mathrm{H}$ subtypes and neutralise group 1 and 2 influenza A viruses, conferring protection to mice and ferrets [43]. Further tests are underway for this broad-spectrum 
antibody as well as more strain-specific monoclonal antibodies, such as the latest candidate that protects against highly pathogenic avian influenza subtype H7 in mice [44]. Additionally, effective prophylaxis was achieved recently using influenza-specific ovine polyclonal antibodies against homologous influenza virus challenge in a mouse model [45]. The study demonstrated full protection independent of a strong innate immune response and may provide another strategy for prophylactic intervention against influenza A virus.

\section{Delivery Routes}

When considering the pulmonary delivery of any drug, it is important to have a detailed knowledge of the healthy respiratory system, and perhaps more importantly, the various diseased states. The respiratory tract contains two main regions, both of which are susceptible to viral infection (figure 3): the conducting airways consisting of the nasal cavity, sinuses, nasopharynx, oropharynx, larynx, trachea and bronchi and the lower respiratory region which includes the bronchioles, alveolar ducts and alveolar sacs.
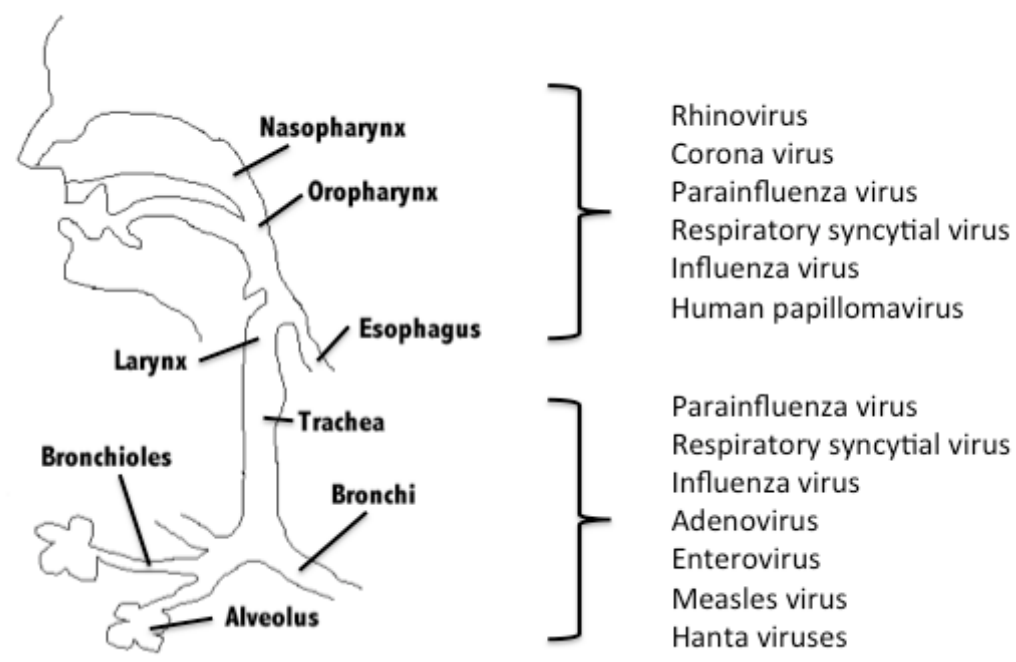

Figure 3. Sites of viral entry and consequent infection in the respiratory tract. A number of viruses enter the conducting airways and cause rhinitis, pharyngitis and laryngitis. Viruses that progress further will infect the lower respiratory tract and can result in tracheitis, bronchitis, pneumonia or disseminated disease. 
Consequently, drug systems have been developed to deliver to these areas via certain entry points, each with varying success. Particular formulations have performed extremely well however, progressing through to clinical trials or approved use and these are outlined below (table 1).

Table 1. Anti-viral and vaccine formulations delivered via the respiratory tract that are currently in use or undergoing clinical trials $\left(^{*}\right)$.

\begin{tabular}{|c|c|c|c|c|c}
\hline Drug/compound & Viral Target & Route & Device & Company & Ref \\
\hline Virazole (ribavirin) & RSV, others & Inhalation & Nebuliser & Valeant Pharmaceuticals & {$[9]$} \\
\hline FluMist & Influenza & Intranasal & Nasal spray & AstraZeneca & {$[58]$} \\
\hline Relenza (zanamir) & Influenza & Inhalation & DPI & Biota, GlaxoSmithKline & {$[73]$} \\
\hline Inavir (laninamivir) & Influenza & Inhalation & DPI & Biota, Daiiichi Sankyo & {$[74]$} \\
\hline TMC353121* & RSV & Inhalation & Nebuliser & Johnson \& Johnson & {$[76]$} \\
\hline ALN-RSV01* & RSV & Inhalation & Nebuliser & Alnylam & {$[80]$} \\
\hline MEDI-534* & RSV, PIV & Intranasal & Nasal spray & Medlmmune LLC & {$[62]$} \\
\hline Carrageenan* & General antiviral & Intranasal & Nasal spray & Marinomed & {$[64]$}
\end{tabular}

\section{Intratracheal instillation}

The main advantage of intratracheal (IT) inoculation has been that it is a direct method that delivers the complete drug dose, bypassing the defenses of the upper respiratory tract. Consequently, it has been used in a number of preclinical animal models to analyse delivery efficacy, toxicity and mechanism of action for treatments against various inflammatory diseases and cystic fibrosis [46-48]. It has also had growing interest in gene transfer studies using adenoviral or lentiviral vector systems $[49,50]$, though the adenoviral backbone has been shown to induce an immune response impeding effective transduction [51].

In regards to viral treatment, there has been limited success with only a handful of studies showing effective outcomes and often only when used in combination with other forms of delivery. DiNapoli and colleagues showed that administering an avian influenza virus vaccine, containing the haemagglutinin protein, by combined IT and intranasal (IN) routes could induce a high level of specific mucosal and serum neutralising antibodies in primates [20]. They later went on 
to show a similar result when inoculating a human primate model with a vaccine against SARS-CoV by combined IT/IN routes [19]. When delivery by the IN route alone was attempted, protective immune responses were not elicited suggesting that the IT route may be effective at delivering further into the lower respiratory tract, though this remains unclear. Another study has demonstrated that a recombinant, attenuated measles vaccine can also elicit robust measles-specific immunity but required an IT inoculation followed by an intramuscular injection [18]. This is most likely due to the fact that the attenuated strain could not replicate efficiently in the respiratory tract following IT inoculation in macaque models [52]. Overall, it suggests that IT administration may not be an effective sole route of delivery, at least for vaccine technologies. This also seems to be the case for cationic carriers as encapsulated nucleic acids demonstrated poor delivery to the lungs when compared to intravenous delivery as seen in mice [53]. Combining this poor outcome with the discomfort and non-uniform drug distribution involved, it makes this procedure less preferable and largely impractical for human therapeutics.

\section{Intranasal administration}

Delivery by the IN route is significantly less invasive than IT administration, but is generally less effective as the compounds can be swallowed or engulfed by the nasal mucosa. Nevertheless, IN delivery of viral therapies has become an important tool, as it can easily deliver anti-virals to affected areas and it provides a safe and needle-free means of vaccination. As vaccines delivered by this route contain live-attenuated virus, they also induce long-lasting mucosal immunity relative to injectable inactivated vaccines. These types of influenza vaccine have long demonstrated an induction of local and systemic antibody production as well as humoural immunity due to the fact that local viral replication mimics the natural infection [54]. Formulations have also shown to produce equivalent serum antibody titers as those delivered by the IM route in rat models [55]. As antigenic variation is an important consideration for influenza vaccination, clinical trials have also been underway using seasonal quadrivalent and potential 
pandemic strains to combat infection via the IN route [56-58]. Other vaccine candidates have been developed with this route in mind. Trials using RSV vaccine by IN delivery have shown some potential, however the required multiple doses and the negative impact of vaccine virus replication have deterred further development to date [17, 59]. Phase II clinical trials with a single dose of live-attenuated PIV have been shown to produce an immunogenic response, however there was no significant difference in the occurrence of adverse events compared to placebo in infant patients [60]. Studies have also investigated the combination of IN delivered live-attenuated RSV and PIV bivalent vaccines and have shown similar responses as monovalent therapies [61]. To overcome this, further trials using the recent MEDI-534 bivalent vaccine have been ongoing in children, though difficulties arising from variable serological responses have been observed $[62,63]$.

The IN route has also been investigated for the delivery of general anti-viral compounds so as to overcome the symptoms generated by a number of respiratory viruses. A prime example of this is the compound carrageenan, which has recently shown promising results against natural cold viruses in clinical trials, significantly reducing viral loads in adult patients [64]. It has also been discovered that the decongestant oxymetazoline, which is used in many intranasal sprays, may also contribute an anti-viral effect against rhinoviruses in patients [65]. While both of these compounds seem to have modest effects, their general mode of action requires multiple, continual doses and so more specialised compounds would be preferable.

Consequently, specific oligonucleotide therapeutics such as siRNA have also been trialled via the direct IN route. One of the first in vivo studies using siRNA directed against SARS-CoV in a rhesus macaque model showed that IN delivery of naked siRNA could reduce viral levels while also abating fever and alveoli damage [66]. Alnylam Pharmaceuticals also used a potent siRNA, termed ALNRSV01, to target a highly conserved region of the RSV nucleocapsid gene. The siRNA was found to be well tolerated, showing limited systemic bioavailability in phase I clinical trials [67], and was then used in phase II trials to treat healthy 
individuals infected with RSV. The IN administration of ALN-RSV01 resulted in the reduction of infected participants by 38\%, however there was no significant reduction in viral load seen compared to placebo [16]. Other groups continue to strive for successful outcomes using in vivo models with IN-delivered siRNA against influenza [23], RSV [24, 68], PIV [24] and other animal viruses such as equine herpesvirus [69]. The latest concepts are also testing combination siRNA therapies such as the silencing of IL-4 expression as well as essential RSV genes to suppress virus growth and related inflammatory responses that exacerbate disease in mouse models [70].

Another specific oligonucleotide therapy demonstrating great promise against disease and viral infection is peptide-bound phosphorodiamidate morpholino oligomers (PPMOs). By interfering with viral protein expression through steric blocking of complementary RNA, PPMOs can specifically target viral proteins essential for replication. The treatment of BALB/c mice prior to RSV infection via the IN route with PPMOs targeting the RSV L gene has shown a significant reduction in viral titres of more than 2 logs in lung tissue [71]. Furthermore, pulmonary inflammation remained attenuated at day 7 post-infection, reducing the severity of disease symptoms. Further development of these compounds and determination of stability, toxicity and effective dose could lead to an effective IN therapy in the future.

\section{Inhalation}

Inhalation through aerosolisation of liquids, powders and vector compounds remains the optimal administration route for pulmonary delivery. Although more costly and hard to measure effective doses, inhalation allows more uniform distribution of compounds with greater penetration. A number of anti-virals have been developed for delivery in this way including the influenza neuraminidase inhibitors, which are the most successful drug target against seasonal and pandemic flu. These include the compounds zanamivir [72, 73] and the more recent laninamivir (Inavir), which produces long-lasting anti-viral 
activity with a single inhalation dose remaining active in the respiratory tract for several days [74]. Similar anti-viral drugs have been developed for RSV but have been less successful. A prime example is the RSV fusion inhibitor RFI-641, which requires inhalation of the nebulised compound for two hours in an African green monkey model before showing a statistically significant log reduction of RSV titres in bronchoalveolar samples [75]. More recently however, the RSV fusion inhibitor 'TMC353121' has shown promising results in both preclinical mice studies [76] and cotton rat models [77] with marked reductions in virus replication and consequential lung inflammation. Following further evaluation, this small molecular inhibitor could prove to have great potential for clinical development.

Anti-viral siRNA therapies have also been administered via inhalation to try and improve the effective delivery of compounds to target areas. Conventional or older methods of aerosolisation can have high shear forces that decrease the efficiency of naked nucleotide delivery. This has generally been overcome by complexing to polyethylenimine [78], or packaging into carrier molecules like biodegradable polyester nanoparticles [79]. Alternatively, newer aerosol devices may also improve delivery via the oral inhalation route. In an attempt to achieve a better response with ALN-RSV01, Alnylam Pharmaceuticals altered their delivery device to an investigational eFlow nebulizer system (PARI Pharma) in a new, small phase IIa trial [80]. Treatment of twenty-four lung transplant patients with three doses of ALN-RSV01 this way showed that the well-tolerated regime resulted in a significant reduction of the incidence of new or progressive clinical sequelae from RSV infection after 90 days. A larger phase IIb clinical trial in lung transplant patients also suggested promising results and further studies for the compound are in discussion.

\section{Challenges for delivery to the virus-infected airway}

Due to the morphology of each region of the respiratory tract, the amount of drug delivered in each area can be quite different. While the lower respiratory 
tract has a large surface area and is directly connected to the systemic circulation via the pulmonary circulation making it an ideal delivery area, factors such as the complex branched nature, the presence of alveolar macrophages and the coverage of pulmonary surfactant can pose substantial obstacles. Moreover, the major components of pulmonary surfactant, that is phospholipids, proteins and mucins, have all been shown to decrease transfection and delivery efficiencies $[81,82]$. Before reaching the lungs, drug compounds must also first travel through the upper airways, overcoming mucociliary and cough clearance mechanisms. Delivery to cells in this passageway is limited due to a smaller surface area, lower regional blood flow and a high capacity to filter out foreign objects, including drugs. Transport is also inhibited as epithelial cells are coated with a mucus layer that traps inhaled substances, while ciliated cells constantly propel the mucus away from the lower respiratory tract.

During viral infection, this can be further compounded by activation of an innate cytokine response. Infection can induce mucosal edema in mice by increasing the vascular endothelial permeability through increased levels of IL-1 $\beta$, IL-6, TNF $\alpha$ and trypsin [83], while the viral-induced inflammasome can become activated, producing airway inflammation and mucus hyperplasia [84, 85]. Basophils and mast cells have also been reported to produce pro-inflammatory cytokines and chemical mediators such as histamine upon infection and this results in further mucus secretion and bronchoconstriction [86, 87]. This cascade of events can create an impermeable barrier to current therapies and so smarter design or alternative delivery routes may need to be considered to effectively deliver to an already infected airway.

\section{Alternative methods for delivery}

\section{Modifications to existing compounds}

As penetration of the airway mucus barrier is required for any kind of drug delivery in the viral-infected lung, closer examination into this layer is necessary. 
Currently, translocation of particulate matter through the barrier is not completely understood, however particle adhesion and diffusion are key indicators of successful delivery. By atomic force microscopy and quantification of penetration velocities, Kirch and colleagues were able to investigate particlemucus interactions and the consequent adhesion properties of some widely used carriers [88]. They discovered that differences in particle adhesion to mucus components were strongly dependent on the particle coating, with polyethylene glycol (PEG) exhibiting mucoinert properties while chitosan coatings showing a strongly increased adhesion to mucus. This would suggest that unlike polysaccharide nanoparticles, which are efficient at mucosal binding in the uninfected lung, small PEG-ylated liposomes may be more likely to avoid being trapped in the mucus layer and instead diffuse to the cells underneath. This has been further demonstrated with IN administration of PEG-coated DNA nanoparticles carrying a plasmid encoding the cystic fibrosis transmembrane conductance regulator gene that resulted in uniform airway distribution and enhanced lung delivery in mouse models [89]. Consequently, polymer composition appears to play a crucial role in the deposition pattern within the respiratory tract and these modifications may improve the penetration of antiviral compounds.

Another alternative might be the use of pulmonary surfactant to improve viralmediated gene transfer or inactivated viral vaccine delivery. Previous work has shown that IT instillation of therapeutic virus in rabbits can spread more effectively into distal lung tissue when combined with surfactant [90]. This was also seen more recently when recombinant adenovirus became ten-fold more effective at reducing tumours in a KRAS mutation-bearing lung cancer mouse model when administered in the presence of surfactant [91]. A further adaptation of this work has also used non-ionic surfactants in combination with mucoactive agents to improve nasal delivery of poorly absorbed hydrophilic compounds in rats [92]. Mucoactive medications include mucolytics that depolymerise polymers of mucin or DNA/actin in pus, mucokinetic agents that increase cough clearance and mucoregulatory medications, which decrease abnormal mucus (reviewed in [93]). These latter compounds especially could be 
useful in reducing mucus hypersecretion, as well as stimulating a potent antiinflammatory effect. This consequence has been suggested for rhinovirus infections, where pre-treatment with the mucoactive drug carbocisteine has shown a reduction in cytokine production in primary tracheal cell culture upon infection [94]. Combining these approaches with efficient drug carriers may then allow diffusion through the otherwise dense barrier and result in more effective delivery of therapeutic agents.

\section{Alternate administration routes}

Due to the numerous physical barriers presented upon delivery via IN, IT or local inhalation and the further obstacles faced by drastic changes in the microenvironment resulting from viral infection, other routes have been proposed as successful workarounds for efficient delivery. Oral agents have been widely used such as amantadine, rimantadine and oseltamivir for influenza, which have worked well to both prevent and treat infection [95-97]. Indeed, oseltamivir (as well as the inhaled alternative zanamivir) is still recommended by the Centre of Disease Control and Prevention for influenza treatment today. Similar trials have also followed suit and include two drugs in clinical trials: pleconaril and T-705 (or favipiravir). Pleconaril is a specific oral inhibitor of picornaviruses and has demonstrated therapeutic efficacy against communityacquired colds caused by rhinoviruses $[98,99]$, while T-705 is an experimental broad-spectrum RNA virus inhibitor [100]. Continuing studies on these compounds may provide further efficacious anti-virals to complement inhaled agents.

Even though the main alternative for inhaled respiratory drugs has been via the oral route, intravenous or systemic administration has also made great strides in facilitating delivery of compounds to target cells in the lung. Initially, the systemic application of naked oligonucleotides was found to be complex as rapid renal clearance, digestion by endogenous nucleases and poor uptake due to inherent qualities resulted in limited therapeutic benefit [101-103]. However 
this was soon remedied by employing similar carrier molecules that were used to deliver via the respiratory tract. Development of these liposomes, polymers and nanoparticles permitted protection from serum exonucleases and excretion, but also were modified to avoid systemic toxicity and travel through the circulatory system to deposit in capillary beds of first-pass organs. As approximately $30 \%$ of all endothelial cells are found in the large surface area of the pulmonary vasculature, many delivery vectors will track to the lung and deliver any therapeutic cargo.

A number of recent studies have also shown that efficient delivery of siRNA can be achieved via intravenous inoculation of lipoplexes. McCaskill and colleagues demonstrated that targeted gene and protein knockdown could be achieved using liposome-encapsulated siRNA directed against the non-essential lamin structural gene in the murine lung [104]. Following a single intravenous dose, $45 \%$ of epithelial cells were positive for uptake showing efficient biodistribution of the therapeutic compound. Similarly, a single dose of DACC siRNA lipoplexes administered by bolus injection or by infusion was shown to sufficiently silence genes expressed in pulmonary endothelial cells such as CD31, Tie-2, VEcadherin, or BMP-R2 [105]. Additionally, incorporation of a surface-modified multifunctional peptide into nanoparticles may further increase delivery to pulmonary epithelia by targeting sialic acid on the cell surface, analogous to the influenza virus [106]. Lastly, siRNA against avian influenza has also been shown to significantly localise to the lungs when linked to a fusion protein based on an anti-haemagglutinin antibody fragment providing evidence of targeted delivery in this manner [107].

Apart from intravenous administration, other systemic routes could be used such as intramuscular administration. However, as they are far removed from the target site, these locations may only serve as a basis for vaccine or gene therapies against viral infection. A prime example of this has shown that intramuscular administration of liposome-DNA complex adjuvant is able to enhance the cross-protective efficacy of influenza A vaccine in mice, generating anti-viral protection for over a year without waning [108]. Furthermore, 
liposome-encapsulated constructs encoding for recombinant antimicrobials have been successfully used to inhibit influenza A virus replication following injection into the muscle [109]. These observations suggest that recombinant proteins and vaccines could be delivered efficiently via the muscle and provide another avenue for virus prevention and treatment.

\section{Conclusion}

There have been a number of significant developments in not only the technologies used to deliver anti-viral drugs to the respiratory tract, but also the formulations, providing biocompatible, biodegradable, non-immunogenic and non-toxic drug compounds that can be delivered efficiently to target cells of the lung. Unfortunately, due to the exacerbation and intensification of a number of physiological and immunological barriers encountered during respiratory virus infection, many superb in vitro or prophylactic therapies have become less effective after viral symptoms have been initiated. Further developments into diffusion and penetrative capability of carrier molecules should facilitate more efficient delivery by the respiratory route during infection, and this is likely to be complemented by clever targeting mechanisms utilised by compound therapies delivered systemically.

\section{Conflicts of Interest}

The authors confirm that this article content has no conflicts of interest.

\section{Acknowledgements}

The authors would like to acknowledge funding from the following agencies: National Health and Medical Research Council of Australia, Cancer Council Queensland, the Griffith Health Institute. 


\section{References}

1. Patton, J.S., J. Bukar, and S. Nagarajan, Inhaled insulin. Adv Drug Deliv Rev, 1999. 35(2-3): p. 235-247.

2. von Mutius, E. and J.M. Drazen, A patient with asthma seeks medical advice in 1828, 1928, and 2012. N Engl J Med, 2012. 366(9): p. 827-34.

3. Freedman, T., Medihaler therapy for bronchial asthma; a new type of aerosol therapy. Postgrad Med, 1956. 20(6): p. 667-73.

4. Green, R.M., et al., Synergism between allergens and viruses and risk of hospital admission with asthma: case-control study. BMJ, 2002. 324(7340): p. 763.

5. O'Donnell, D.R. and P.J. Openshaw, Anaphylactic sensitization to aeroantigen during respiratory virus infection. Clin Exp Allergy, 1998. 28(12): p. 1501-8.

6. Mounts, P., K.V. Shah, and H. Kashima, Viral etiology of juvenile- and adultonset squamous papilloma of the larynx. Proc Natl Acad Sci U S A, 1982. 79(17): p. 5425-9.

7. Balazic, J., A. Masera, and M. Poljak, Sudden death caused by laryngeal papillomatosis. Acta Otolaryngol Suppl, 1997. 527: p. 111-3.

8. Gillison, M.L., et al., Evidence for a causal association between human papillomavirus and a subset of head and neck cancers. J Natl Cancer Inst, 2000. 92(9): p. 709-20.

9. Ribavirin aerosol approved for severe cases of RSV in infants and young children. FDA Drug Bull, 1986. 16(1): p. 7.

10. Da Dalt, L., et al., Oseltamivir-resistant pandemic (H1N1) 2009 treated with nebulized zanamivir. Emerg Infect Dis, 2010. 16(11): p. 1813-5.

11. Foster, J.M., et al., The reliability and patient acceptability of the SmartTrack device: a new electronic monitor and reminder device for metered dose inhalers. J Asthma, 2012. 49(6): p. 657-62.

12. Ermers, M.J.J., et al., The effect of high dose inhaled corticosteroids on wheeze in infants after respiratory syncytial virus infection: randomised double blind placebo controlled trial. BMJ, 2009. 338.

13. Adi, H., P.M. Young, and D. Traini, Co-deposition of a triple therapy drug formulation for the treatment of chronic obstructive pulmonary disease using solution-based pressurised metered dose inhalers. J Pharm Pharmacol, 2012. 64(9): p. 1245-53.

14. Coates, M.S., et al., Effect of design on the performance of a dry powder inhaler using computational fluid dynamics. Part 1: Grid structure and mouthpiece length. J Pharm Sci, 2004. 93(11): p. 2863-76.

15. Cheer, S.M. and A.J. Wagstaff, Spotlight on zanamivir in influenza. Am J Respir Med, 2002. 1(2): p. 147-52.

16. DeVincenzo, J., et al., A randomized, double-blind, placebo-controlled study of an RNAi-based therapy directed against respiratory syncytial virus. Proc Natl Acad Sci U S A, 2010. 107(19): p. 8800-5.

17. Wright, P.F., et al., Evaluation of a live, cold-passaged, temperaturesensitive, respiratory syncytial virus vaccine candidate in infancy. J Infect Dis, 2000. 182(5): p. 1331-42. 
18. Bolton, D.L., et al., Priming T-cell responses with recombinant measles vaccine vector in a heterologous prime-boost setting in non-human primates. Vaccine, 2012. 30(41): p. 5991-8.

19. DiNapoli, J.M., et al., Delivery to the lower respiratory tract is required for effective immunization with Newcastle disease virus-vectored vaccines intended for humans. Vaccine, 2009. 27(10): p. 1530-9.

20. DiNapoli, J.M., et al., Immunization of primates with a Newcastle disease virus-vectored vaccine via the respiratory tract induces a high titer of serum neutralizing antibodies against highly pathogenic avian influenza virus. J Virol, 2007. 81(21): p. 11560-8.

21. Sun, L., et al., Adenovirus-mediated in vivo silencing of anaphylatoxin receptor C5aR. J Biomed Biotechnol, 2006. 2006(4): p. 28945.

22. Niven, R.W. and H. Schreier, Nebulization of liposomes. I. Effects of lipid composition. Pharm Res, 1990. 7(11): p. 1127-33.

23. Tompkins, S.M., et al., Protection against lethal influenza virus challenge by RNA interference in vivo. Proc Natl Acad Sci U S A, 2004. 101(23): p. 86826.

24. Bitko, V., et al., Inhibition of respiratory viruses by nasally administered siRNA. Nat Med, 2005. 11(1): p. 50-5.

25. Tai, W., et al., Multistrain influenza protection induced by a nanoparticulate mucosal immunotherapeutic. Mucosal Immunol, 2011. 4(2): p. 197-207.

26. Zhang, T., et al., Antisense oligonucleotides targeting the RNA binding region of the NP gene inhibit replication of highly pathogenic avian influenza virus H5N1. Int Immunopharmacol, 2011. 11(12): p. 2057-61.

27. Wong, J.P., et al., Activation of toll-like receptor signaling pathway for protection against influenza virus infection. Vaccine, 2009. 27(25-26): p. 3481-3.

28. Tang, Y., et al., Development and evaluation of a dry powder formulation of liposome-encapsulated oseltamivir phosphate for inhalation. Drug Deliv, 2013.

29. Benoit, A., et al., Effects of alveolar macrophage depletion on liposomal vaccine protection against respiratory syncytial virus (RSV). Clin Exp Immunol, 2006. 145(1): p. 147-54.

30. Zhao, J., et al., Evasion by stealth: inefficient immune activation underlies poor $T$ cell response and severe disease in SARS-CoV-infected mice. PLoS Pathog, 2009. 5(10): p. e1000636.

31. Illum, L., Nasal drug delivery - recent developments and future prospects. J Control Release, 2012. 161(2): p. 254-63.

32. Zhang, W., et al., Inhibition of respiratory syncytial virus infection with intranasal siRNA nanoparticles targeting the viral NS1 gene. Nat Med, 2005. 11(1): p. 56-62.

33. Kong, X., et al., Respiratory syncytial virus infection in Fischer 344 rats is attenuated by short interfering RNA against the RSV-NS1 gene. Genet Vaccines Ther, 2007. 5: p. 4.

34. Hui, Z., et al., Studies on polyamidoamine dendrimers as efficient gene delivery vector. J Biomater Appl, 2008. 22(6): p. 527-44.

35. Kukowska-Latallo, J.F., et al., Intravascular and endobronchial DNA delivery to murine lung tissue using a novel, nonviral vector. Hum Gene Ther, 2000. 11(10): p. 1385-95. 
36. Landers, J.J., et al., Prevention of influenza pneumonitis by sialic Acidconjugated dendritic polymers. J Infect Dis, 2002. 186(9): p. 1222-30.

37. Luo, K., et al., Peptide dendrimers as efficient and biocompatible gene delivery vectors: Synthesis and in vitro characterization. J Control Release, 2011. 155(1): p. 77-87.

38. Johnson, S., et al., Development of a humanized monoclonal antibody (MEDI-493) with potent in vitro and in vivo activity against respiratory syncytial virus. J Infect Dis, 1997. 176(5): p. 1215-24.

39. Wu, H., et al., Development of motavizumab, an ultra-potent antibody for the prevention of respiratory syncytial virus infection in the upper and lower respiratory tract. J Mol Biol, 2007. 368(3): p. 652-65.

40. Huang, K., et al., Respiratory syncytial virus-neutralizing monoclonal antibodies motavizumab and palivizumab inhibit fusion. J Virol, 2010. 84(16): p. 8132-40.

41. Ramilo, 0., et al., Motavizumab Treatment of Infants Hospitalized With Respiratory Syncytial Virus Infection Does Not Decrease Viral Load or Severity of Illness. Pediatr Infect Dis J, 2013.

42. Okuno, Y., et al., A common neutralizing epitope conserved between the hemagglutinins of influenza A virus H1 and H2 strains. J Virol, 1993. 67(5): p. 2552-8.

43. Corti, D., et al., A neutralizing antibody selected from plasma cells that binds to group 1 and group 2 influenza A hemagglutinins. Science, 2011. 333(6044): p. 850-6.

44. He, F., et al., Effective intranasal therapeutics and prophylactics with monoclonal antibody against lethal infection of H7N7 influenza virus. Antiviral Res, 2013. 100(1): p. 207-14.

45. Rinaldi, C., et al., Modulation of innate immune responses by influenzaspecific ovine polyclonal antibodies used for prophylaxis. PLoS One, 2014. 9(2): p. e89674.

46. Moschos, S.A., et al., Lung delivery studies using siRNA conjugated to TAT(48-60) and penetratin reveal peptide induced reduction in gene expression and induction of innate immunity. Bioconjug Chem, 2007. 18(5): p. 1450-9.

47. Li, T. and H.G. Folkesson, RNA interference for alpha-ENaC inhibits rat lung fluid absorption in vivo. Am J Physiol Lung Cell Mol Physiol, 2006. 290(4): p. L649-L660.

48. Messer, M.P., et al., Silencing of fas, fas-associated via death domain, or caspase 3 differentially affects lung inflammation, apoptosis, and development of trauma-induced septic acute lung injury. Shock, 2013. 39(1): p. 19-27.

49. Carlon, M., et al., Efficient gene transfer into the mouse lung by fetal intratracheal injection of rAAV2/6.2. Mol Ther, 2010. 18(12): p. 2130-8.

50. DuPage, M., A.L. Dooley, and T. Jacks, Conditional mouse lung cancer models using adenoviral or lentiviral delivery of Cre recombinase. Nat Protoc, 2009. 4(7): p. 1064-72.

51. Van Ginkel, F.W., et al., Intratracheal gene delivery with adenoviral vector induces elevated systemic IgG and mucosal IgA antibodies to adenovirus and beta-galactosidase. Hum Gene Ther, 1995. 6(7): p. 895-903. 
52. de Vries, R.D., et al., In vivo tropism of attenuated and pathogenic measles virus expressing green fluorescent protein in macaques. J Virol, 2010. 84(9): p. 4714-24.

53. Ge, Q., et al., Inhibition of influenza virus production in virus-infected mice by RNA interference. Proc Natl Acad Sci U S A, 2004. 101(23): p. 8676-81.

54. Zangwill, K.M., et al., Prospective, randomized, placebo-controlled evaluation of the safety and immunogenicity of three lots of intranasal trivalent influenza vaccine among young children. Pediatr Infect Dis J, 2001. 20(8): p. 740-6.

55. Garmise, R.J., H.F. Staats, and A.J. Hickey, Novel dry powder preparations of whole inactivated influenza virus for nasal vaccination. AAPS PharmSciTech, 2007. 8(4): p. E81.

56. Talaat, K.R., et al., An open-label phase I trial of a live attenuated H2N2 influenza virus vaccine in healthy adults. Influenza Other Respir Viruses, 2013. 7(1): p. 66-73.

57. Block, S.L., et al., A randomized, double-blind noninferiority study of quadrivalent live attenuated influenza vaccine in adults. Vaccine, 2011. 29(50): p. 9391-7.

58. Nichol, K.L., et al., Effectiveness of live, attenuated intranasal influenza virus vaccine in healthy, working adults: a randomized controlled trial. JAMA, 1999. 282(2): p. 137-44.

59. Malkin, E., et al., Safety and immunogenicity of a live attenuated RSV vaccine in healthy RSV-seronegative children 5 to 24 months of age. PLoS One, 2013. 8(10): p. e77104.

60. Belshe, R.B., et al., Phase 2 evaluation of parainfluenza type 3 cold passage mutant 45 live attenuated vaccine in healthy children 6-18 months old. J Infect Dis, 2004. 189(3): p. 462-70.

61. Belshe, R.B., et al., Evaluation of combined live, attenuated respiratory syncytial virus and parainfluenza 3 virus vaccines in infants and young children. J Infect Dis, 2004. 190(12): p. 2096-103.

62. Bernstein, D.I., et al., Phase 1 study of the safety and immunogenicity of a live, attenuated respiratory syncytial virus and parainfluenza virus type 3 vaccine in seronegative children. Pediatr Infect Dis J, 2012. 31(2): p. 10914.

63. Yang, C.F., et al., Implication of respiratory syncytial virus (RSV) F transgene sequence heterogeneity observed in Phase 1 evaluation of MEDI-534, a live attenuated parainfluenza type 3 vectored RSV vaccine. Vaccine, 2013. 31(26): p. 2822-7.

64. Ludwig, M., et al., Efficacy of a carrageenan nasal spray in patients with common cold: a randomized controlled trial. Respir Res, 2013. 14: p. 124.

65. Winther, B., et al., Decreased rhinovirus shedding after intranasal oxymetazoline application in adults with induced colds compared with intranasal saline. Am J Rhinol Allergy, 2010. 24(5): p. 374-7.

66. Li, B.J., et al., Using siRNA in prophylactic and therapeutic regimens against SARS coronavirus in Rhesus macaque. Nat Med, 2005. 11(9): p. 944-51.

67. DeVincenzo, J., et al., Evaluation of the safety, tolerability and pharmacokinetics of ALN-RSV01, a novel RNAi antiviral therapeutic directed against respiratory syncytial virus (RSV). Antiviral Res, 2008. 77(3): p. 225-31. 
68. Akinc, A., et al., A combinatorial library of lipid-like materials for delivery of RNAi therapeutics. Nat Biotechnol, 2008. 26(5): p. 561-9.

69. Fulton, A., et al., Effective treatment of respiratory alphaherpesvirus infection using RNA interference. PLoS One, 2009. 4(1): p. e4118.

70. Khaitov, M.R., et al., siRNAs targeted to $I L-4$ and $R S V$ reduce airway inflammation in a mouse model of virus-induced asthma exacerbation. Hum Gene Ther, 2014.

71. Lai, S.H., et al., Inhibition of respiratory syncytial virus infections with morpholino oligomers in cell cultures and in mice. Mol Ther, 2008. 16(6): p. 1120-8.

72. Lalezari, J., et al., Zanamivir for the treatment of influenza $A$ and $B$ infection in high-risk patients: a pooled analysis of randomized controlled trials. Arch Intern Med, 2001. 161(2): p. 212-7.

73. Makela, M.J., et al., Clinical efficacy and safety of the orally inhaled neuraminidase inhibitor zanamivir in the treatment of influenza: a randomized, double-blind, placebo-controlled European study. J Infect, 2000. 40(1): p. 42-8.

74. Watanabe, A., et al., Long-acting neuraminidase inhibitor laninamivir octanoate versus oseltamivir for treatment of influenza: A double-blind, randomized, noninferiority clinical trial. Clin Infect Dis, 2010. 51(10): p. 1167-75.

75. Weiss, W.J., et al., Inhalation efficacy of RFI-641 in an African green monkey model of RSV infection. J Med Primatol, 2003. 32(2): p. 82-8.

76. Olszewska, W., et al., Antiviral and lung protective activity of a novel respiratory syncytial virus fusion inhibitor in a mouse model. Eur Respir J, 2011. 38(2): p. 401-8.

77. Bonfanti, J.F., et al., Selection of a respiratory syncytial virus fusion inhibitor clinical candidate. 2. Discovery of a morpholinopropylaminobenzimidazole derivative (TMC353121). J Med Chem, 2008. 51(4): p. 875-96.

78. Rudolph, C., et al., Aerosolized nanogram quantities of plasmid DNA mediate highly efficient gene delivery to mouse airway epithelium. Mol Ther, 2005. 12(3): p. 493-501.

79. Nguyen, J., et al., Fast degrading polyesters as siRNA nano-carriers for pulmonary gene therapy. J Control Release, 2008. 132(3): p. 243-51.

80. Zamora, M.R., et al., RNA interference therapy in lung transplant patients infected with respiratory syncytial virus. Am J Respir Crit Care Med, 2011. 183(4): p. 531-8.

81. Duncan, J.E., J.A. Whitsett, and A.D. Horowitz, Pulmonary surfactant inhibits cationic liposome-mediated gene delivery to respiratory epithelial cells in vitro. Hum Gene Ther, 1997. 8(4): p. 431-8.

82. Rosenecker, J., et al., Interaction of bronchoalveolar lavage fluid with polyplexes and lipoplexes: analysing the role of proteins and glycoproteins. J Gene Med, 2003. 5(1): p. 49-60.

83. Wang, S., et al., Influenza virus-cytokine-protease cycle in the pathogenesis of vascular hyperpermeability in severe influenza. J Infect Dis, 2010. 202(7): p. 991-1001.

84. Chang, Y.J., et al., Innate lymphoid cells mediate influenza-induced airway hyper-reactivity independently of adaptive immunity. Nat Immunol, 2011. 12(7): p. 631-8. 
85. Stokes, K.L., et al., The respiratory syncytial virus fusion protein and neutrophils mediate the airway mucin response to pathogenic respiratory syncytial virus infection. J Virol, 2013. 87(18): p. 10070-82.

86. Chonmaitree, T., et al., Role of interferon in leukocyte histamine release caused by common respiratory viruses. J Infect Dis, 1988. 157(1): p. 12732.

87. Hosoda, M., et al., Effects of rhinovirus infection on histamine and cytokine production by cell lines from human mast cells and basophils. J Immunol, 2002. 169(3): p. 1482-91.

88. Kirch, J., et al., Optical tweezers reveal relationship between microstructure and nanoparticle penetration of pulmonary mucus. Proc Natl Acad Sci U S A, 2012. 109(45): p. 18355-60.

89. Suk, J.S., et al., Lung gene therapy with highly compacted DNA nanoparticles that overcome the mucus barrier. J Control Release, 2014. 178: p. 8-17.

90. Jobe, A.H., et al., Surfactant enhances adenovirus-mediated gene expression in rabbit lungs. Gene Ther, 1996. 3(9): p. 775-9.

91. Fukazawa, T., et al., Targeting KRAS mutation-bearing lung cancer in vivo by pulmonary surfactant-adenovirus-mediated gene transfer. Anticancer Res, 2010.30(12): p. 4925-35.

92. Matsuyama, T., et al., Enhancement of nasal absorption of large molecular weight compounds by combination of mucolytic agent and nonionic surfactant. J Control Release, 2006. 110(2): p. 347-52.

93. Balsamo, R., L. Lanata, and C.G. Egan, Mucoactive drugs. Eur Respir Rev, 2010. 19(116): p. 127-33.

94. Yasuda, H., et al., Carbocisteine inhibits rhinovirus infection in human tracheal epithelial cells. Eur Respir J, 2006. 28(1): p. 51-8.

95. Hayden, F.G., et al., Use of the selective oral neuraminidase inhibitor oseltamivir to prevent influenza. N Engl J Med, 1999. 341(18): p. 1336-43.

96. Monto, A.S., et al., Safety and efficacy of long-term use of rimantadine for prophylaxis of type A influenza in nursing homes. Antimicrob Agents Chemother, 1995. 39(10): p. 2224-8.

97. Sears, S.D. and M.L. Clements, Protective efficacy of low-dose amantadine in adults challenged with wild-type influenza $A$ virus. Antimicrob Agents Chemother, 1987. 31(10): p. 1470-3.

98. Hayden, F.G., et al., Efficacy and safety of oral pleconaril for treatment of colds due to picornaviruses in adults: results of 2 double-blind, randomized, placebo-controlled trials. Clin Infect Dis, 2003. 36(12): p. 1523-32.

99. Pevear, D.C., et al., Relationship of pleconaril susceptibility and clinical outcomes in treatment of common colds caused by rhinoviruses. Antimicrob Agents Chemother, 2005. 49(11): p. 4492-9.

100. Furuta, Y., et al., T-705 (favipiravir) and related compounds: Novel broadspectrum inhibitors of RNA viral infections. Antiviral Res, 2009. 82(3): p. 95-102.

101. Urban-Klein, B., et al., RNAi-mediated gene-targeting through systemic application of polyethylenimine (PEI)-complexed siRNA in vivo. Gene Ther, 2005. 12(5): p. 461-6.

102. Gao, S., et al., The effect of chemical modification and nanoparticle formulation on stability and biodistribution of siRNA in mice. Mol Ther, 2009. 17(7): p. 1225-33. 
103. Watts, J.K., G.F. Deleavey, and M.J. Damha, Chemically modified siRNA: tools and applications. Drug Discov Today, 2008. 13(19-20): p. 842-55.

104. McCaskill, J., et al., Efficient Biodistribution and Gene Silencing in the Lung epithelium via Intravenous Liposomal Delivery of siRNA. Mol Ther Nucleic Acids, 2013. 2: p. e96.

105. Fehring, V., et al., Delivery of Therapeutic siRNA to the Lung Endothelium via Novel Lipoplex Formulation DACC. Mol Ther, 2014. 22(4): p. 811-20.

106. Kusumoto, K., et al., Lipid envelope-type nanoparticle incorporating a multifunctional peptide for systemic siRNA delivery to the pulmonary endothelium. ACS Nano, 2013. 7(9): p. 7534-41.

107. Zhang, T., et al., Antisense oligonucleotide inhibits avian influenza virus H5N1 replication by single chain antibody delivery system. Vaccine, 2011. 29(8): p. 1558-64.

108. Dong, L., et al., Cationic liposome-DNA complexes (CLDC) adjuvant enhances the immunogenicity and cross-protective efficacy of a prepandemic influenza A H5N1 vaccine in mice. Vaccine, 2012. 30(2): p. 25464.

109. Li, W., et al., Construction of eukaryotic expression vector with mBD1-mBD3 fusion genes and exploring its activity against influenza $A$ virus. Viruses, 2014. 6(3): p. 1237-52. 